\title{
Facile separation of PEGylated liposomes enabled by anti-PEG scFv
}

Wenjing Tang ${ }^{1,2, \#, ~ Z u i ~ Z h a n g ~}{ }^{2, \#}$, Cheng Li ${ }^{3, \#, ~ Y u x i u ~ C h u ' ~}{ }^{2}$, Jun Qian" ${ }^{1}$, Tianlei Ying ${ }^{3, *}$, Weiyue $\mathrm{Lu}^{1, *}$, Changyou Zhan ${ }^{1,2, *}$

${ }^{1}$ MOE Key Laboratory of Smart Drug Delivery, School of Pharmacy \& State Key Laboratory of Molecular Engineering of Polymers, Fudan University, Shanghai, 201203 P.R. China

${ }^{2}$ Department of Pharmacology, School of Basic Medical Sciences \& Center of Medical Research and Innovation, Shanghai Pudong Hospital, Fudan University, Shanghai, 200032 P.R. China

${ }^{3}$ Key Laboratory of Medical Molecular Virology (MOE/NHC/CAMS), School of Basic Medical Sciences, Fudan University, Shanghai, 200032 PR China.

\#These authors contribute equally to this work.

*To whom correspondence should be addressed. Tel: 86-21-54237379. E-mail: cyzhan@fudan.edu.cn (CZ), wylu@shmu.edu.cn (WL) or tlying@fudan.edu.cn (TY)

\section{Materials and methods}

\section{Animals and Cells}

Adult male SD rats weighting $200 \sim 220$ g, male BALB/C mice aging $6 \sim 8$ weeks and male C57BL/6 mice weighting $18 \sim 20 \mathrm{~g}$ were purchased from Shanghai SLAC Laboratory Animal Co. Ltd (Shanghai, China) and raised under SPF conditions. All animal experiments were proceeded under the guidelines evaluated and approved by the Ethics Committee of Fudan University. MC-38 cells were purchased from Shanghai Institute of Cell Biology, and maintained in Dulbecco's Modified Eagle Medium (DMEM, Hyclone) supplemented with 10\% fetal bovine serum (FBS, Gibco) at $37{ }^{\circ} \mathrm{C}$ under $5 \% \mathrm{CO}_{2}$.

\section{Human serum}

Human serum samples were accessed from Pudong Hospital, Fudan University, Shanghai. The use of human samples was certified by the Ethics Committee of the Fudan University and informed consent documents were accessed from each health donor.

\section{Materials and reagents}


Materials for liposomes preparation including hydrogenated soy phosphatidylcholine (HSPC), cholesterol and $\mathrm{mPEG}_{2000}$-DSPE were bought from AVT Pharmaceutical Co., Ltd. (Shanghai, China). Fluorescent dye DiI (1,1'-Dioctadecyl-3,3,3',3'-tetramethylindocarbo-cyanine perchlorate, 42364) and Sephadex ${ }^{\circledR}$ G-50 (G5080) were purchased from Sigma (St. Louis, MO). HisSep Ni-NTA agarose resin 6FF (20503ES10), Ampicillin (60203ES10) and Polymyxin B Sulfate (60242ES03) were acquired from YEASEN Biotech Co., Ltd (Shanghai, China). HRP conjugated anti-6X His tag antibody (ab1187), goat anti-rat IgM mu chain (HRP) (ab97180) and goat anti-human IgM mu chain (HRP) (ab97205) were purchased from Abcam (Cambridge, MA). Gradient Precast Protein Gels 4 20\% (4561093) were purchased from BioRad Co., Ltd (Shanghai, China). Tryptone (A650217) and Yeast Extract (A100850) were from Sangon Biotech (Shanghai, China). Doxorubicin (MB1087-S) and Daunorubicin (MB1074) were purchased from Meilunbio Co., Ltd. (Dalian, China). SPE column (68003) was from DIKMA Co., Ltd. (Beijing, China). Pierce ${ }^{\mathrm{TM}}$ BCA Protein Assay Kit (23225) and 1-Step ${ }^{\mathrm{TM}}$ ABTS Substrate (37615) were from Thermo Scientific Co., Ltd. (Waltham, MA). Fast Silver Stain Kit (P0017S) and TMB Substrate (P0209) were from Beyotime Biotechnology Co., Ltd. (Shanghai, China).

\section{Expression and characterization of PEG-scFv (b1)}

In our previous work, $\mathrm{PEG}-\mathrm{scFv}$ was prepared and used to ameliorate $\mathrm{ABC}$ effect and separate protein corona ${ }^{23}$. Here we introduced PEG-scFv (b1) through a site-specific mutagenesis to enhance the affinity of PEG-scFv to PEG unit as described ${ }^{24}$. The affinity maturation plasmid PEG-scFv was constructed and cloned into the competent E. coli (TOP10). After culturing overnight, several monoclonals were picked up and amplified. The expression E. coli were collected by centrifugation at $4{ }^{\circ} \mathrm{C}, 5000 \times \mathrm{g}$ for $30 \mathrm{~min}$. The pellets were resuspended with Lysis Buffer ( $0.5 \mathrm{M} \mathrm{NaCl}$ and $0.1 \mathrm{M}$ polymyxin B sulfate), and the supernatant was obtained after centrifugation at $4{ }^{\circ} \mathrm{C}, 12000 \times \mathrm{g}$ for $10 \mathrm{~min}$. PEG-scFv (b1) was purified through Ni-NTA agarose resin and eluted with Elution Buffer (phosphate buffered saline PBS with $250 \mathrm{mM}$ imidazole, $\mathrm{pH}$ adjusted to 8.0). The eluted fractions were concentrated at $12000 \times g$ and washed with chilled PBS for at least three times, until the storage buffer was completely replaced. BCA protein assay kit was used to detect the concentration of PEG-scFv (b1). Gradient polyacrylamide gel $(4 \% \sim 20 \%)$ and fast sliver staining kit were applied to validate the purity of PEG-scFv (b1).

The affinity of PEG-scFv (b1) binding to PEG was characterized by ELISA kit. PEGylated liposomes (sLip) were diluted with PBS and immobilized on 96-well microplate with $50 \mu \mathrm{g}$ 
HSPC per well at $4{ }^{\circ} \mathrm{C}$ overnight. $\mathrm{mPEG}_{2000}$-DSPE ( $2 \mu \mathrm{g}$ per well) was diluted in absolute ethanol and coated on microplates, respectively. After blocking with 5\% BSA in PBS, serial dilutions of PEG-scFv (b1) in 10\% BALB/c mouse plasma or PBS were incubated for $1.5 \mathrm{~h}$ at $37{ }^{\circ} \mathrm{C}$. CR301x-1 (His tagged-scFv without activity for PEG) was set as control. HRP conjugated anti-6X His antibody (1:5000 dilution) was used and detected by TMB substrate at OD $450 \mathrm{~nm}$.

\section{Preparation and characterization of PEGylated liposomes}

sLip were prepared by thin film hydration and extrusion method as previously described ${ }^{25}$. Briefly, HSPC (9.58 mg), Cholesterol (3.19 mg) and $\mathrm{mPEG}_{2000}$-DSPE (3.19 mg) were fully dissolved in chloroform and vacuum distillated to remove organic solvent until a thin film was formed. The residual chloroform was removed under vacuum overnight. The dried film was hydrated with $1 \mathrm{~mL}$ saline at $60^{\circ} \mathrm{C}$ and extruded through polycarbonate membranes with pore diameters of $400 \mathrm{~nm}, 200 \mathrm{~nm}$ and $100 \mathrm{~nm}$, each for $8 \sim 10$ times. Then 1\% and 2\% PEGylated liposomes were prepared by reducing the $\mathrm{mPEG}_{2000}$-DSPE to $0.64 \mathrm{mg}$ and $1.28 \mathrm{mg}$ per $\mathrm{mL}$. DiI labeled liposomes (sLip/DiI) were prepared following the same procedure of 5\% sLip except by adding $100 \mu \mathrm{g} \mathrm{mL}^{-1}$ of DiI to the mixture of membrane materials in chloroform. The unloaded DiI was removed by G50 column. Doxorubicin encapsulated liposomes (sLip/DOX, with $9.58 \mathrm{mg} \mathrm{mL}^{-1} \mathrm{HSPC}$ and $1 \mathrm{mg} \mathrm{mL}^{-1} \mathrm{DOX}$ ) were prepared by traditional ammonium sulfate gradient method as reported ${ }^{26}$. Liposomes were 20 times diluted with deionized water (DW), to be characterized for size and zeta potential by a Zetasizer Nano ZS90 (Malvern Instruments, Malvern, UK). Doxorubicin loading efficiency was measured using reversed-phase high performance liquid chromatography (rp-HPLC).

\section{The affinity precipitation of PEGylated liposomes by PEG-scFv (b1)}

To study the sLip/DiI precipitation induced by the affinity of PEG-scFv (b1), $10 \mu \mathrm{L} 1 \%, 2 \%$ and 5\% PEGylated sLip/DiI were mixed with $50 \mu \mathrm{L}$ BALB/C mouse plasma, then mixtures were incubated with $0 \sim 50 \mu \mathrm{g}$ PEG-scFv (b1) for $30 \mathrm{~min}$. Supernatant was collected by centrifugation at $4{ }^{\circ} \mathrm{C}, 2000 \times g$ for $10 \mathrm{~min}$. sLip/DiI in supernatant were quantified at Ex/Em $535 / 585 \mathrm{~nm}$.

To optimize the centrifuge speed for separation, 5\% PEGylated sLip/DiI $(10 \mu \mathrm{L})$ were mixed with $50 \mu \mathrm{L}$ BALB/C mouse plasma, and $40 \mu \mathrm{g}$ PEG-scFv (b1) was used to precipitate sLip/DiI. The supernatant was collected by centrifugation at $4{ }^{\circ} \mathrm{C}, 100 \sim 2000 \times g$ for $10 \mathrm{~min}$, then the sedimentation ratio (\%) of sLip/DiI was quantified by fluorescence intensity at $535 / 585 \mathrm{~nm}$. In 
order to identify the amount of PEG-scFv (b1) absorbed on sLip, $10 \mu \mathrm{L}$ sLip were incubated with $0 \sim 50 \mu \mathrm{g}$ PEG-scFv (b1) in PBS. The co-precipitated sLip-scFv were collected by centrifugation and detected by BCA kit.

\section{The separation of sLip/DOX and free DOX by SPE}

The SPE column was activated by cold $\mathrm{CH}_{3} \mathrm{OH}$, washed with chilled DW and saturated by precooled fetal bovine serum (FBS). Here $10 \mu \mathrm{L}$ sLip/DOX (9.58 $\mathrm{mg} \mathrm{mL}^{-1}$ HSPC) were added with $50 \mu \mathrm{L} \mathrm{BALB/C}$ mouse plasma as well as free DOX (final concentration 1, 2 or $10 \mu \mathrm{g}$ $\left.\mathrm{mL}^{-1}\right)$. The samples were layered onto the SPE columns, the flow through was collected as Elute 1. Elute 2 and Elute 3 were collected by adding $50 \mu \mathrm{L}$ cold FBS and $250 \mu \mathrm{L}$ chilled DW, respectively. Elute1, 2 and 3 were mixed as the sLip/DOX fraction and detected by rp-HPLC. DW was added to wash the column, then methanol (containing $0.5 \%$ formic acid) was used to collect the free DOX component. The detect limit of rp-HPLC here is $5 \mathrm{ng}(\mathrm{S} / \mathrm{N}>10$ set as effective data) for DOX. When free DOX was undetectable by rp-HPLC, it was calculated by formula: Conc $_{\text {free DOX }}=$ Conc $_{\text {total DOX }}-$ Conc $_{\text {sLip/DOX. }}$.

\section{The integrity characterization of the sLip/DOX}

For morphology characterization, $\operatorname{sLip} / \mathrm{DOX}\left(9.58 \mathrm{mg} \mathrm{mL}^{-1} \mathrm{HSPC}, 10 \mu \mathrm{L}\right)$ were incubated with PEG-scFv $(2 \mu \mathrm{g}$ or $20 \mu \mathrm{g})$ at $4{ }^{\circ} \mathrm{C}$ for $10 \mathrm{~min}$, sLip/DOX in PBS were set as control. PEG8000 (final concentration $1 \mathrm{mg} \mathrm{mL}^{-1}$ ) was added to the mixtures of sLip/DOX and PEG-scFv to recover the conjugated liposomes to individuals. All samples were 10 times diluted with chilled PBS and captured by Cryo-EM with 50000 magnifications.

To investigate the DOX leakage during PEG-scFv based method, we set three groups for DOX detection: (1) sLip/DOX; (2) sLip + $10 \mu \mathrm{g} \mathrm{mL}^{-1} \mathrm{DOX}$; (3) sLip/DOX $+10 \mu \mathrm{g} \mathrm{mL}^{-1} \mathrm{DOX}$ in $50 \%$ BALB/C mice plasma. Samples were added with $40 \mu \mathrm{g}$ PEG-scFv (b1), and incubated at $4{ }^{\circ} \mathrm{C}$ for $30 \mathrm{~min}$. The supernatant was collected by centrifugation at $4{ }^{\circ} \mathrm{C}, 2000 \times g$ for $10 \mathrm{~min}$. The supernatant of sLip/DOX group was then treated with SPE to obtain liposomal DOX fraction as aforementioned in SPE method. Here LC/MS-MS-8060 (SHIMADZU) which has a detection limit of $0.5 \mathrm{pg}$ DOX $(\mathrm{S} / \mathrm{N}>10)$ was implied to quantify the concentration of DOX. The supernatants of sLip + DOX group and sLip/DOX + DOX group were added with $100 \mu \mathrm{L}$ daunorubicin $\left(10 \mu \mathrm{g} \mathrm{mL}^{-1}\right.$ in $\left.\mathrm{CH}_{3} \mathrm{OH}\right)$ and $400 \mu \mathrm{L} \mathrm{CHCl}_{3}$ successively. After extracting, chloroform layer was collected and evaporated in fume hoods. rp-HPLC (40\% acetylene) was used to determine the concentration of doxorubicin in supernatant.

\section{In vitro separation of sLip/DOX and free DOX}


sLip $\left(9.58 \mathrm{mg} \mathrm{mL}^{-1} \mathrm{HSPC}, 10 \mu \mathrm{L}\right)$ and $50 \mu \mathrm{L} \mathrm{BALB} / \mathrm{C}$ mouse plasma were mixed, and free DOX (final concentration $1 \mu \mathrm{g} \mathrm{mL}^{-1}$ ) was added to improve the detection limit of rp-HPLC. The mixture were placed at room temperature for $15 \mathrm{~min}$, then incubated with $40 \mu \mathrm{g}$ PEG-scFv (b1) at $4{ }^{\circ} \mathrm{C}$ or room temperature for $30 \mathrm{~min}$. The supernatant was acquired by centrifugation at $500 \times g, 1000 \times g$ or $2000 \times g$ for $10 \mathrm{~min}$. The content of doxorubicin in supernatant was determined by rp-HPLC refer to the previous section for specific methods.

sLip $(10 \mu \mathrm{L}$ ) were added with $50 \mu \mathrm{L}$ BALB/C mouse plasma and $20 \mu \mathrm{L}$ free DOX (with final concentration of 1,2 or $10 \mu \mathrm{g} \mathrm{mL}^{-1}$ ). After incubating at room temperature for $15 \mathrm{~min}$, the mixtures were added with $40 \mu \mathrm{g}$ PEG-scFv (b1) and incubated for further $30 \mathrm{~min}$ at $4{ }^{\circ} \mathrm{C}$. The supernatant was collected by centrifugation at $4{ }^{\circ} \mathrm{C}, 2000 \times \mathrm{g}$ for $10 \mathrm{~min}$ and quantified by rpHPLC. The mixtures of $10 \mu \mathrm{L}$ sLip with 1,2 or $10 \mu \mathrm{g} \mathrm{mL}-1$ free DOX in $50 \%$ BALB/C mouse plasma were separated with SPE, respectively. The concentration of sLip/DOX and free DOX were detected by rp-HPLC.

\section{In vivo separation of sLip/DOX and free DOX}

Male SD rats weighting 200 220 g were applied to evaluate the DOX released from sLip/DOX in vivo. SD rats $(\mathrm{n}=5)$ were intravenously injected with $\mathrm{sLip} / \mathrm{DOX}\left(5 \mathrm{mg} \mathrm{kg}^{-1}\right)$, and blood (anticoagulant with EDTA·2Na) was sampled at $10 \mathrm{~min}, 30 \mathrm{~min}, 1 \mathrm{~h}, 2 \mathrm{~h}, 4 \mathrm{~h}, 8 \mathrm{~h}, 12 \mathrm{~h}$ and $24 \mathrm{~h}$ after injection. The plasma was collected by centrifugation at $4{ }^{\circ} \mathrm{C}, 1200 \times g$ for $8 \mathrm{~min}$. The volume of $60 \mu \mathrm{L}$ plasma sample was added with $40 \mu \mathrm{L}$ PEG-scFv (b1) $\left(1 \mathrm{mg} \mathrm{mL}^{-1}\right)$, then incubated at $4{ }^{\circ} \mathrm{C}$ for $30 \mathrm{~min}$. The supernatant containing free DOX was collected by centrifugation at $2000 \times g$ for $10 \mathrm{~min}$. For SPE separation, $100 \mu \mathrm{L}$ plasma sample was loaded onto column and manipulated as aforementioned. Extraction of DOX and detection by rpHPLC was applied as in vitro separation method.

\section{Anti-PEG IgM detection in serum}

SD rats weighting $200 \sim 220 \mathrm{~g}$ were intravenously stimulated with sLip $\left(0.23 \mathrm{mg} \mathrm{kg}^{-1}\right)$, and the serum at $6^{\text {th }}$ day after stimulation was sampled, which was supposed to contain large amount of anti-PEG IgM. ELISA 96 wells microplate were coated $\mathrm{mPEG}_{2000}$-DSPE in EtOH with $2 \mu \mathrm{g}$ per well. After blocking with 5\% BSA, serial dilutions of serum were added and incubated at $37^{\circ} \mathrm{C}$ for $1 \mathrm{~h}$. HRP conjugated anti-rat IgM (1:5000 diluted in 0.1\% BSA-PBS) was added and incubated for another $1 \mathrm{~h}$. ABTS substrate was used to detect the binding ability of anti-PEG IgM to PEG at OD $405 \mathrm{~nm}$.

For human serum, serial serum dilutions of patient 135 (P135) and patient 137 (P137) were 
added in the $\mathrm{mPEG}_{2000}$-DSPE coated microplates and incubated for $1 \mathrm{~h}$ at $37^{\circ} \mathrm{C}$. Secondary antibody HRP-conjugated anti-human IgM (1:5000) was applied to detect the pre-existing antiPEG IgM in human serum.

\section{Statistical analysis}

All statistical analyses were calculated by GraphPad Prism 8.0.1 (San Diego CA, USA, www.graphpad.com). Two-way ANOVA (multiple comparisons) of grouped analyses was adopted to examine differences in comparison of two or more groups. $\mathrm{p}<0.05$ was considered as significant differences. 